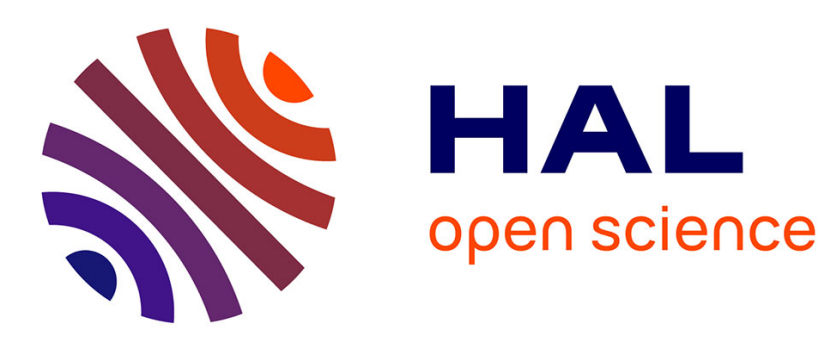

\title{
Far-Field Wavefront Control of Nonlinear Luminescence in Disordered Gold Metasurfaces
}

Gauthier Roubaud, Pierre Bondareff, Giorgio Volpe, Sylvain Gigan, Sébastien Bidault, Samuel Grésillon

\section{> To cite this version:}

Gauthier Roubaud, Pierre Bondareff, Giorgio Volpe, Sylvain Gigan, Sébastien Bidault, et al.. FarField Wavefront Control of Nonlinear Luminescence in Disordered Gold Metasurfaces. Nano Letters, 2020, 20 (5), pp.3291-3298. 10.1021/acs.nanolett.0c00089 . hal-02995818

\section{HAL Id: hal-02995818 https://hal.science/hal-02995818}

Submitted on 9 Nov 2020

HAL is a multi-disciplinary open access archive for the deposit and dissemination of scientific research documents, whether they are published or not. The documents may come from teaching and research institutions in France or abroad, or from public or private research centers.
L'archive ouverte pluridisciplinaire HAL, est destinée au dépôt et à la diffusion de documents scientifiques de niveau recherche, publiés ou non, émanant des établissements d'enseignement et de recherche français ou étrangers, des laboratoires publics ou privés. 


\title{
Far-field Wavefront Control of Nonlinear
}

\section{Luminescence in Disordered Gold Metasurfaces}

\author{
Gauthier Roubaud, ${ }^{\dagger}$ Pierre Bondareff, ${ }^{\dagger}$ Giorgio Volpe, ${ }^{\ddagger} \uparrow$ Sylvain Gigan, ${ }^{\ddagger}$ \\ Sébastien Bidault, ${ }^{* \dagger}$ and Samuel Grésillon ${ }^{*, \dagger}$ \\ $\dagger$ Institut Langevin, ESPCI Paris, PSL University, CNRS, Sorbonne Université, 1 rue \\ Jussieu, F-75005 Paris, France \\ $\ddagger$ Laboratoire Kastler Brossel, Sorbonne Université, École Normale Supérieure-PSL \\ University, CNRS, Collège de France, 24 rue Lhomond, 75005 Paris, France \\ \Current address: Department of Chemistry, University College London, 20 Gordon Street, \\ London, WC1H OAJ, UK \\ E-mail: sebastien.bidault@espci.fr; samuel.gresillon@espci.fr
}

\begin{abstract}
We demonstrate the local optimization of nonlinear luminescence from disordered gold metasurfaces by shaping the phase of a femtosecond excitation. This process is enabled by the far-field wavefront control of plasmonic modes delocalized over the sample surface, leading to a coherent enhancement of sub-wavelength electric fields. In practice, the increase in nonlinear luminescence is strongly sensitive to both the nanometerscale morphology and to the level of structural complexity of the gold metasurface. We typically observe a two-order of magnitude enhancement of the luminescence signal for an optimized excitation wavefront compared to a random one. These results demonstrate how disordered metasurfaces made of randomly coupled plasmonic resonators, together with wavefront shaping, provide numerous degrees of freedom to program locally optimized nonlinear responses and optical hotspots.
\end{abstract}


Keywords: Wavefront shaping, plasmonics, metasurfaces, nonlinear luminescence, disordered media

Disorder in complex media has recently undergone a paradigm shift with the ability to control multiply-scattered light using wavefront shaping: from a major drawback when considering aberrations and attenuation through diffusion, disorder becomes a powerful tool to engineer wave propagation in space ${ }^{1-3}$ and time. ${ }^{4-8}$ A scattering medium can be programmed as an adaptable lens, ${ }^{1-3,9-11}$ a pulse shaper ${ }^{7,12}$ or an optical processor. ${ }^{13}$ On the other hand, plasmonic resonators, with their ability to confine and enhance electromagnetic fields at deep subwavelength scales, ${ }^{14,15}$ provide complementary degrees of freedom in controlling the propagation of light.

Designing metallic antennas and surfaces with coupled plasmonic modes indeed allows farfield phase modulation to tune local electric fields. ${ }^{16-24}$ It is, for instance, possible to turn on or off the field enhancement in a nanoparticle dimer ${ }^{17}$ or to switch between several hotspots in a more complex nanoantenna. ${ }^{16,18,19,24}$ However, the use of simple resonators strongly limits the number and position of hotspots that can be controlled. Deterministic 2D surfaces can be engineered to provide extra degrees of freedom for wavefront shaping ${ }^{21}$ but their fabrication by focused ion beam milling is difficult and costly; and their programmability is very sensitive to fabrication errors. ${ }^{22}$ As an alternative, we propose to use disordered gold metasurfaces that are easy and cost-effective to fabricate and which feature both strong local field enhancements, or hotspots, at numerous positions of the sample, as well as delocalized plasmonic modes ${ }^{14,25-29}$ that can be coherently modulated. ${ }^{30-33}$ Furthermore, disordered or aperiodic metasurfaces made of coupled resonators exhibit stronger local field enhancements than comparable regular arrays. ${ }^{34,35}$ We demonstrate here that optical hotspots in disordered plasmonic surfaces are coherently optimized using wavefront shaping; proving that electric near-fields can be engineered using a far-field phase modulation.

Disordered gold films are fabricated by depositing gradually a nanometer-scale layer of gold 
on a glass substrate: partial dewetting of the metal leads to the formation of random islands that can exhibit electrical percolation if a given filling fraction of gold, $f f$, is reached on the surface (called percolation threshold, see supporting information for more details). ${ }^{36}$ These rough metallic surfaces have been extensively used as substrates for surface-enhanced spectroscopy ${ }^{37-39}$ as they feature strong local field enhancements ${ }^{14,29}$ for a spectral range that is significantly broader than comparable regular arrays of plasmonic resonators. ${ }^{40,41}$ In practice, the modulation of optical hotspots is monitored in-situ by measuring nonlinear photoluminescence (NPL) from gold under a femtosecond pulsed excitation. While the physical origin of NPL in gold nanostructures is still under debate, ${ }^{42-44}$ this technique has been shown to provide a direct estimation of near-field enhancements when there is one hotspot per diffraction-limited area. ${ }^{17,45}$ Interestingly, photo-emission electron microscopy measurements on disordered gold metasurfaces demonstrated that the number of hotspots per wavelength squared is between 1 and 2 for a given excitation wavelength and polarization. ${ }^{29}$ Fig. 1 -a describes the employed optical setup: the wavefront of a linearly-polarized femtosecond pulsed laser is shaped by a spatial light modulator (SLM). An inverted microscope with a highnumerical aperture $(N A=1.4)$ conjugates the SLM with the plane of a gold metasurface over a given area (later referred to as wavefront-shaped area). The femtosecond excitation is collimated after the microscope objective and the size of the SLM macropixels are chosen such that their image on the sample surface is comparable to the diffraction limit. Finally, nonlinear luminescence emitted by the gold surface is imaged on a high-sensitivity sCMOS camera. This optical setup is therefore optimized to tune independently the phase of the incoming wave on the sample surface for a homogeneous excitation, while measuring the NPL intensity. However, taking into account the limited numerical aperture of the microscope, tuning the excitation phase at scales similar to the diffraction limit will necessarily induce small fluctuations of the excitation intensity on the sample surface. We demonstrate below that the influence of this effect on the ability to optimize nonlinear luminescence emitted by disordered gold metasurfaces using phase-only wavefront shaping is negligible when the size 


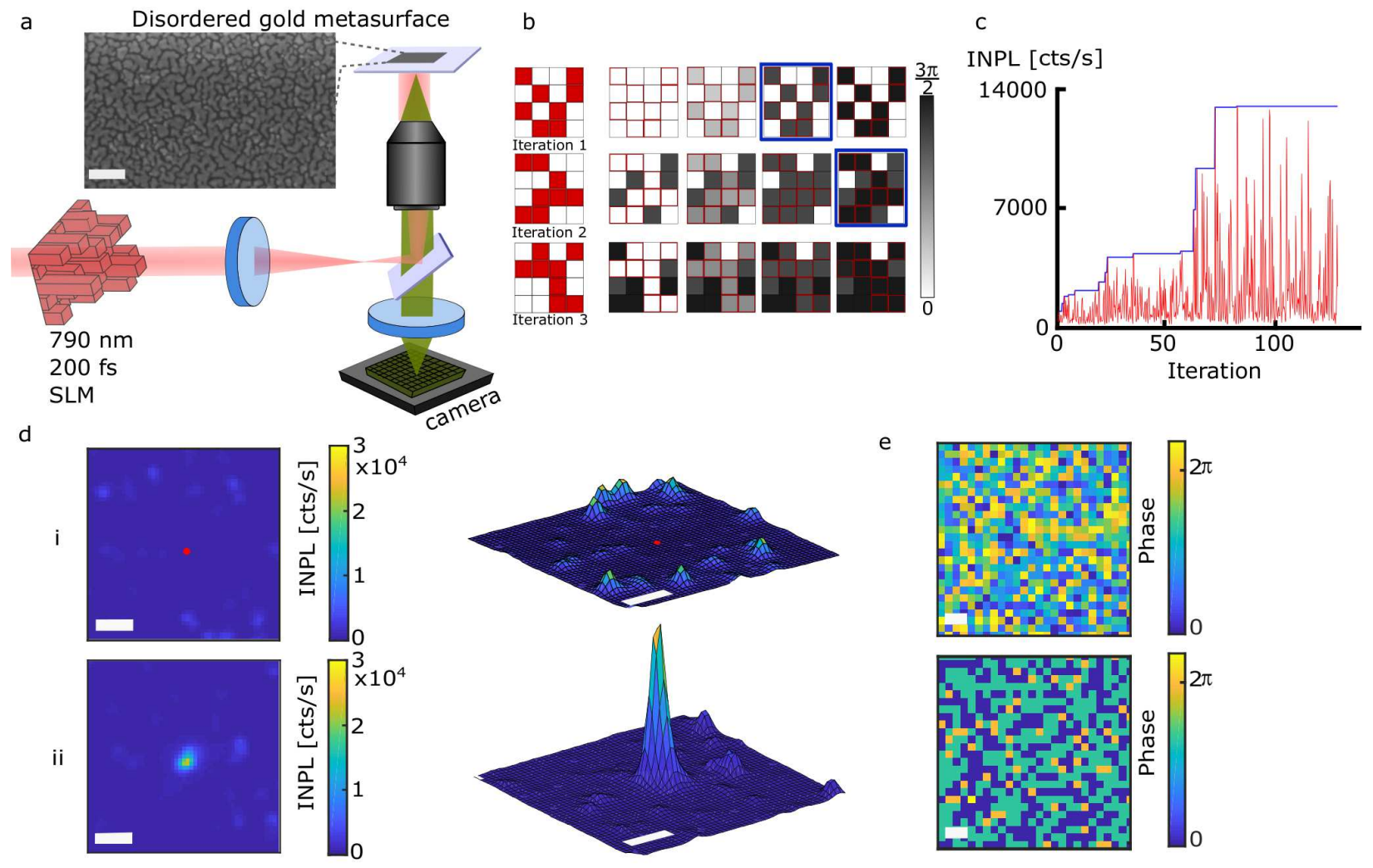

Figure 1: Wavefront-shaping to optimize nonlinear luminescence in disordered gold metasurfaces (a) Experimental setup: a phase-modulated femtosecond excitation is conjugated with the plane of a disordered gold surface in an inverted microscope (insert: typical scanning electron microscopy image, scale bar is $100 \mathrm{~nm}$ ). Nonlinear photoluminescence intensity (INPL) is then imaged on a high-sensitivity camera after spectral filtering. (b) Principle of the random iterative algorithm: half of the pixels of an initial random phase pattern are randomly selected. During the first iteration, these pixels have their phase modulated over four values $0, \pi / 2, \pi$ and $3 \pi / 2$. If one phase provides a higher NPL intensity in the center of the wavefront-shaped area than the initial random phase-mask, it is then selected as the new optimized phase pattern (outlined in blue). In the following iterations, half of the pixels are randomly chosen and a similar selection is performed. If the phase patterns of iteration $n$ do not provide a higher NPL signal, the wavefront of the iteration $n-1$ is retrieved for the $n+1$ iteration. (c) Typical evolution of the NPL intensity in the center of the wavefront-shaped area during optimization (data for each phase value of each iteration in red and evolution of the maximum intensity in blue). Examples of 2D and 3D NPL images (d) and corresponding phase-masks (e) before (i) and after (ii) optimization for a gold metasurface close to the percolation threshold (scale bars are $1 \mu \mathrm{m}$ ). In this case, the wavefront-shaped area is a 20 $\mu \mathrm{m}$ square. The red circles mark the center of the wavefront-shaped area in which nonlinear luminescence is optimized during the random iteration algorithm.

of the wavefront-shaped area on the sample is significantly larger than the diffraction limit.

An iterative random optimization algorithm ${ }^{9,10}$ is used to increase the nonlinear lumines- 
cence signal in the center of the wavefront-shaped area, as described in Fig. 1-b. Starting from an initially random phase pattern, half of the pixels of the SLM are randomly selected to define a modulation mask (represented in red in Fig. 1-b). This mask is tuned over 4 phases between 0 and $3 \pi / 2$. If, for one of these 4 phases, the strongest NPL intensity in the center of the wavefront-shaped area is larger than with the initial random phase pattern, this modified wavefront is selected (outlined in blue in Fig. 1-b). For the second iteration, a randomly chosen half of the pixels of this new phase pattern are again modulated over 4 phases: if a larger NPL intensity is found, a new optimized wavefront is selected for the next iteration; while, if the signal decreases, the next iteration is performed on the last phase pattern that provided the largest NPL intensity. Fig. 1-c shows a typical increase of the NPL intensity during the optimization algorithm: a maximum is found after several tens of iterations with most iterations leading to a signal reduction. The duration of the optimization procedure is limited to $15 \mathrm{~min}$ (130 iterations) in order to avoid mechanical instabilities in the optical setup (see supporting information - SI - for more details). This means that the optimization procedure may not be optimal, not only because random algorithms cannot reach a global maximum, ${ }^{10}$ but also because of the limited experimental duration and because the algorithm only considers 4 possible phases. However, in more than $75 \%$ of the measurements, the highest observed NPL signal is obtained before the 100th iteration.

Fig. 1-d shows an example of NPL images before and after wavefront optimization for a plasmonic metasurface close to the percolation threshold $(f f=0.64)$, as well as the corresponding initial and final phase masks. In this case, the wavefront-shaped area is a $20 \mu \mathrm{m}$ square, which is about 4 times the interaction length observed for delocalized plasmonic modes in gold surfaces with a filling fraction close to the percolation threshold. ${ }^{33}$ We observe a significant increase of the nonlinear luminescence specifically at the chosen position of the sample (center of the wavefront-shaped area) with a factor of 91 . We can also note that other positions of the sample typically exhibit a small reduction of the NPL intensity, demonstrating the complex interplay of local optical hotspots and delocalized modes that in- 
fluence the nonlinear emission process. A general trend in these experiments is the increased homogeneity of the final phase mask compared to the initial random one. This indicates that the optimization procedure is efficient and comes partially from the low number of phases that are generated (4 different phases between 0 and $3 \pi / 2$ ). It might also be linked to the limited number of delocalized modes that coherently interfere to enhance the NPL signal. It is important to stress that the optimized nonlinear luminescence signal corresponds to a diffraction-limited area (see Fig. S1 in the SI for more details): since, in plasmonic nanostructures, large luminescence intensities originate from strong local field enhancements, ${ }^{42,46}$ this result indicates that far-field wavefront shaping indeed controls and enhances near-field optical hotspots in disordered gold metasurfaces.

The local electric field enhancements ${ }^{14,27,29}$ as well as the interaction lengths of delocalized plasmonic modes ${ }^{33}$ are sensitive to the filling fraction of noble metals in disordered surfaces. Therefore, we performed several tens of iterative random optimizations for different thicknesses of deposited gold as shown on Fig. 2 (maintaining a $20 \times 20 \mu \mathrm{m}^{2}$ square as the wavefront-shaped area). These data demonstrate unequivocally the influence of the nanoscale morphology of gold islands on the ability to enhance nonlinear luminescence using far-field wavefront shaping. Indeed, Fig. 2-a presents the distributions of NPL signals before and after optimization for a disordered metasurface far from percolation $(f f=0.29)$, one close to percolation $(f f=0.64)$ and one significantly above it $(f f=0.76)$ : we observe that both the initial and final intensities are significantly larger close to percolation. By fitting these distributions with log-normal probability density functions, it is possible to infer average initial and final NPL intensities as well as standard errors. This allows the definition of an average enhancement factor, whose dependence with respect to the gold filling fraction is plotted in Fig. 2-b alongside the average NPL intensities after optimization. Close to percolation, the final nonlinear signals are more than one order of magnitude larger than far from it and also correspond to the largest enhancement factors, reaching nearly two orders of magnitude when $f f=0.64$. 

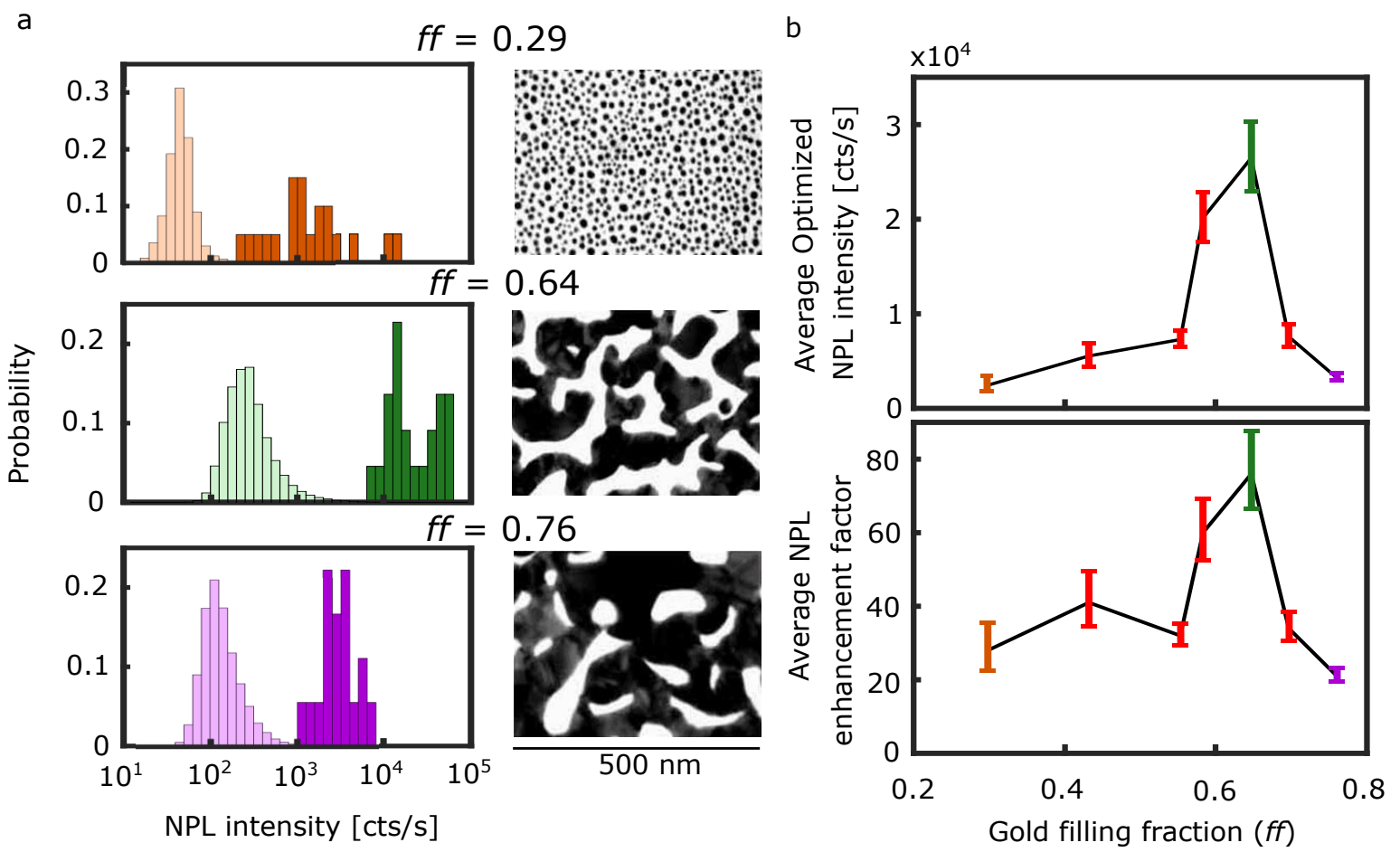

Figure 2: Influence of the nanometer-scale morphology of the disordered metasurface. (a) Semi-logarithmic distributions of NPL intensities before (light color bars) and after (dark color bars) 20 optimization procedures for three different filling fractions of gold $(f f=0.29$, orange bars; $f f=0.64$, green bars; $f f=0.76$, purple bars - typical corresponding transmission electron microscopy images are shown to the right). The initial NPL intensities are estimated over $10^{4}$ pixels around the optimized position (in the center of the wavefront-shaped area). The final NPL intensities are estimated as the largest value in the 9 pixels around the center of the wavefront-shaped area after optimization. (b) Optimized nonlinear luminescence intensities and average NPL enhancement factors as a function of the gold filling fraction. The average enhancement factors are estimated using the means of the log-normal distributions of Fig. 2-a. The error bars for the optimized nonlinear luminescence intensities are the standard errors of the log-normal distributions. The error bars for the enhancement factors are the standard errors of the optimized intensity divided by the mean initial intensity (for which the standard error is negligible).

It is interesting to note that significant enhancement factors are also observed at low filling fractions where the metasurface features a high density of isolated gold islands. In this case, plasmon polaritons do not propagate on continuous metallic nanostructures but through coupled plasmonic modes as studied in 1D arrays of regular nanoparticles. ${ }^{4-49}$ Our measurements demonstrate that wavefront shaping can be exploited in extended 2D arrays of isolated plasmonic resonators. However, Fig. 2-b clearly shows that the added structural 
complexity and disorder of percolated films, which combine continuous metallic particles and coupled plasmonic modes, leads to a significant gain in NPL intensities and enhancement factors. This is in agreement with previous studies that demonstrated stronger local modulations of the electric field ${ }^{27,29}$ and plasmonic modes delocalized over longer distances around percolation $(5-6 \mu \mathrm{m}$ interaction lengths at $f f=0.64$ compared to $2-3 \mu \mathrm{m}$ at low filling fractions). ${ }^{33}$

The experimental data provided in Fig. 1 and Fig. 2 indicate that tuning the phase when exciting delocalized modes in disordered plasmonic metasurfaces, several microns away from the area of interest, leads to a coherent increase of a chosen optical hotspot and, therefore, of the corresponding NPL intensity. This phenomenon should be strongly sensitive to the number of accessible delocalized modes and therefore to the size of the wavefront-shaped area. ${ }^{33}$ We investigate in Fig. 3 the sensitivity of the random optimization procedure with respect to the size of the wavefront-shaped area (averaged over 20 realizations), for a fixed gold filling fraction close to percolation. We observe two regimes: a first increase of the luminescence signal when the size of the wavefront-shaped area is tuned between $2 \mu \mathrm{m}$ and $6 \mu \mathrm{m}$; followed by a reduction and a second increase from $10 \mu \mathrm{m}$ to $40 \mu \mathrm{m}$, above which the signal tends to saturate. In practice, the origin of the large NPL intensities with small and large wavefront-shaped areas are significantly different: when the modified phase-mask is comparable in size with the diffraction limit at $790 \mathrm{~nm}$ (Fig. 3-a-i), the iterative optimization of the luminescence signal originates largely from fluctuations of the excitation intensity due to spherical aberrations (grey area in Fig. 3-b); while, when the wavefront-shaped area is significantly larger than the diffraction limit (Fig. 3-a-ii and iii), the random algorithm essentially exploits phase-only modulations (white area in Fig. 3-b).

Considering the numerical aperture of the optical setup and the wavelength of the femtosecond laser, the diffraction limit is of the order of $700 \mathrm{~nm}$; comparable in size with the image, on the sample plane, of the macropixels of the SLM on which the phase of the incoming field is modulated (square of typically $360 \times 360 \mathrm{~nm}^{2}$ ). In practice, a sharp phase 


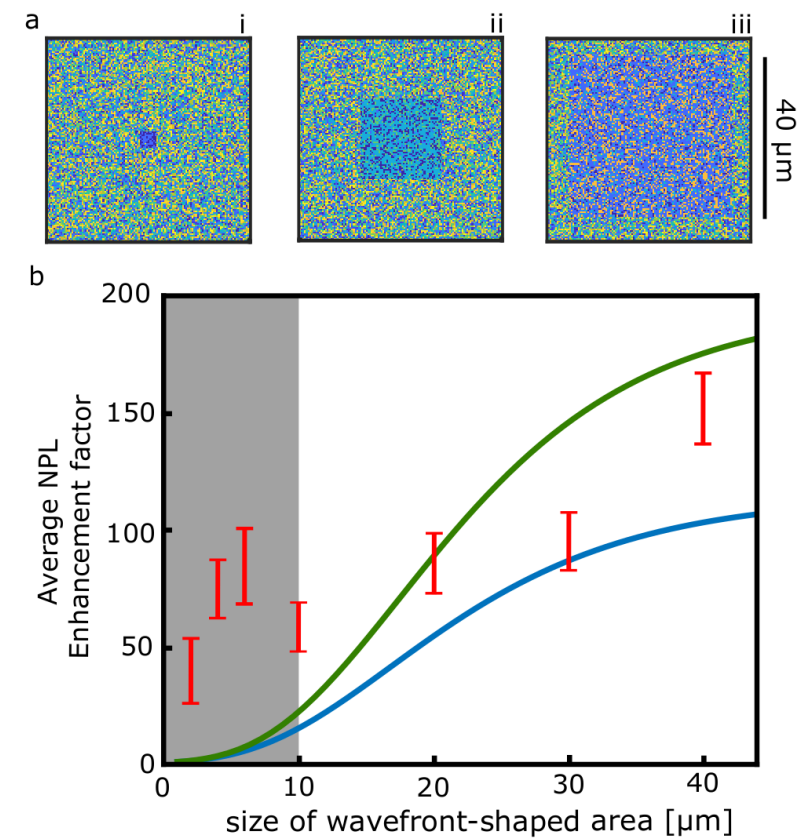

Figure 3: Influence of the size of the wavefront-shaped area on the NPL enhancement factor. (a) Examples of optimized phase masks when the wavefront-shaped area is a $4 \mu \mathrm{m}$ (i), 20 $\mu \mathrm{m}$ (ii) or $40 \mu \mathrm{m}$ (iii) square. (b) Evolution of the average enhancement factors (measured over 20 realizations) as a function of the size of the wavefront-shaped area, for a fixed filling fraction close to the percolation threshold. The error bars are estimated similarly to Fig. 2-b. The gray area highlights the experimental conditions for which fluctuations of the excitation intensity play an important role on the measured NPL enhancement factor. The solid lines are theoretical estimations of the enhancement factors as a function of the size of the wavefront-shaped area using a simple phenomenological model with two parameters: the interaction length $L$ of the delocalized plasmonic modes in the metasurface and the coupling efficiency $\eta$ to these delocalized modes. Blue line: $L=5 \mu \mathrm{m} / \eta=0.011$. Green line: $L=5 \mu \mathrm{m} / \eta=0.013$.

difference between two consecutive macropixels will weakly diffract the excitation field in the plane of the sample, leading to low intensity oscillations at the interface between pixels over a typical distance of several times the diffraction limit. Therefore, the image of the phase-only SLM on the disordered gold surface is not a pure phase-mask but features small fluctuations of the electromagnetic field amplitude. These fluctuations are strongly reduced when the macropixel size is increased. But the size at which they disappear is comparable to the interaction length of delocalized modes on the plasmonic metasurface, making the optimization procedure inherently a complex mix of amplitude and phase modulations. Importantly, the optimization algorithm is random and selects any phase mask that increases 
the NPL intensity, either thanks to constructive interferences between delocalized plasmonic modes or between electric fields diffracted by several macropixels leading to a higher excitation intensity in the center of the wavefront-shaped area. Therefore the NPL intensity may increase without involving delocalized plasmonic modes.

Hence, we experimentally verify that, for a small wavefront-shaped area, the dominant effect is a local increase of the excitation intensity, while this not the case for large wavefront-shaped area. For this purpose, we measure the two-photon fluorescence image of a homogeneous polymer film doped with fluorescein, using phase patterns that were selected by the random algorithm on the gold metasurface. Fig. 4-a shows typical images of the gold NPL and fluorescein TPF intensities when the square wavefront-shaped area is $4 \mu \mathrm{m}$ wide. It also provides distributions of the NPL and TPF signals measured on the optimized area after 18 independent iterative procedures, compared to the distributions of initial NPL and TPF signals, for the same size of the wavefront-shaped area. On the fluorescent sample, we systematically observe an increased TPF intensity in the center of the wavefront-shaped area after optimization with an average enhancement of 4 . Considering the different nonlinear intensity dependence of the TPF (quadratic) and gold NPL (2.8 power dependence) signals (see Fig. S2), this corresponds to an increased nonlinear luminescence on the gold surface of a factor 7 . It is interesting to note that the random algorithm can be directly implemented with the homogenous fluorescent film providing average TPF enhancements of 6 (see Fig. S3), further evidencing the issue of intensity fluctuations on the optimization process. However, this process cannot fully account for the enhancement observed in Fig. 3-b for a $4 \mu \mathrm{m}$ wavefront-shaped area, meaning that the optimization algorithm mixes intensity fluctuations and phase modulations to enhance the NPL signal.

Fig. 4-b provides a similar comparison between gold NPL and fluorescein TPF signals for 24 optimization procedures with a $20 \mu \mathrm{m}$ wide phase pattern. In about $75 \%$ of the measurements, the TPF intensity in the optimized area is comparable with the rest of the sample. In practice, the TPF intensity distribution indicates a moderate average enhancement of 


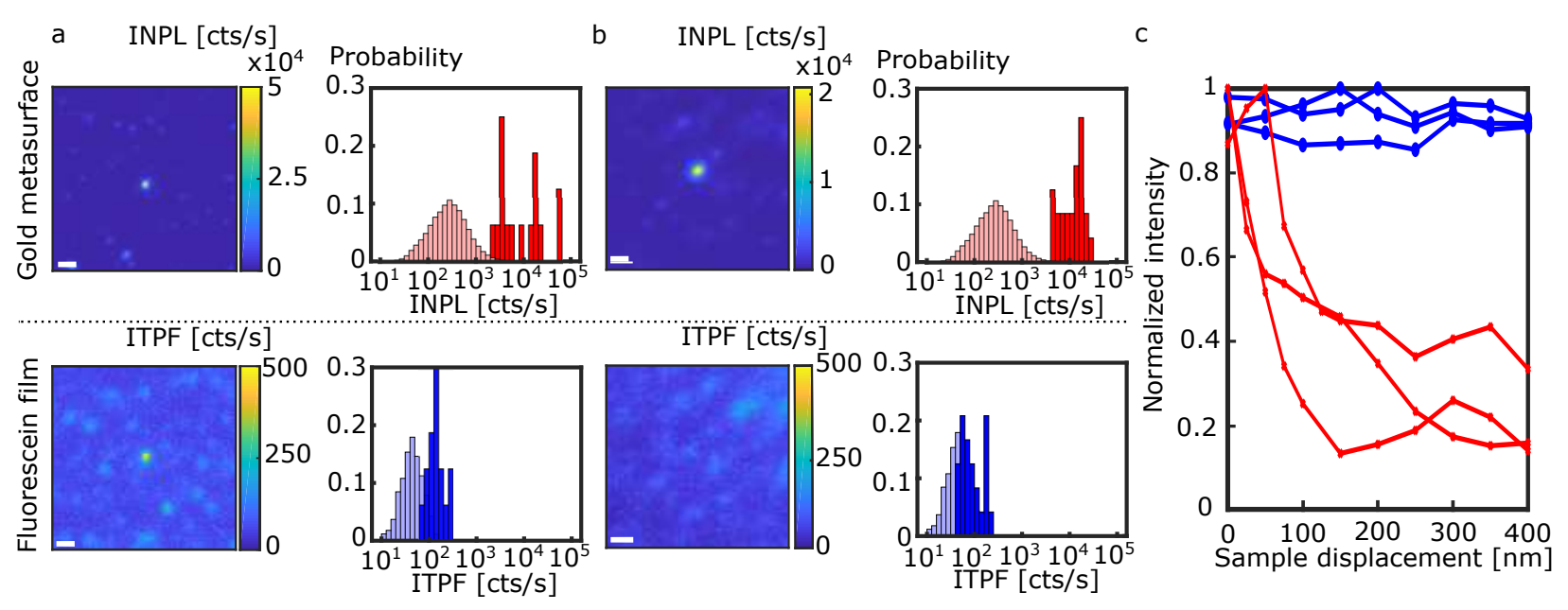

Figure 4: Influence of excitation intensity fluctuations on the wavefront optimization of the NPL signal. Typical images of the NPL intensity on a disordered gold metasurface after optimization (top left) and of the TPF signal of a homogeneous fluorescent sample using the optimized phase pattern (bottom left), as well as distributions of the NPL (red data, top right) and TPF signals (blue data, bottom right) before (light color bars) and after (dark color bars) iterative optimization for two sizes of the wavefront-shaped area: $4 \mu \mathrm{m}$ (a) and $20 \mu \mathrm{m}$ (b). Scale bars are $1 \mu \mathrm{m}$. (c) Three examples of the evolution of the NPL (red data) and TPF (blue data) signals, in the center of the wavefront-shaped area after optimization, when the sample is moved while the phase pattern is kept constant.

1.5, corresponding to less than a factor 2 for the NPL signal. The enhancements of NPL intensities observed on the gold metasurface in Fig. 3-b, for large wavefront-shaped areas, are therefore essentially due to phase modulations. This can be understood when considering that when half of the pixels of a large phase-mask are randomly selected by the algorithm for each iteration, the probability that these pixels are at a diffraction-limited distance from the center of the wavefront-shaped area is weak. For small phase-masks, most selected pixels can influence the intensity at the center of the wavefront-shaped area. To further evidence that the optimized gold NPL intensity for large phase-masks is due to a complex phase pattern selected for a given morphology of the disordered metasurface, we measure the evolution of the signal as we displace the sample using a piezoelectric stage. After optimization, the gold NPL signal typically drops by $80 \%$ over a few hundred nanometers, while an optimized fluorescein TPF signal is independent of the sample position. This further evidences that intensity fluctuations do not influence significantly the NPL optimization process for large 
wavefront-shaped areas. It also confirms the subwavelength origin of the optimized NPL intensities.

The data of Fig. 4 indicate that the experimental enhancement factors measured in Fig. 3 could be slightly overestimated compared to a hypothetical phase-only wavefront shaping process during which the excitation intensity remains perfectly homogeneous. However, the limited duration of the iterative process and the limited number of phases in the algorithm suggest, on the contrary, underestimated enhancement factors. It is therefore interesting to investigate whether the measured values of Fig. 3 qualitatively agree with the considered underlying phase-only optimization mechanism: electric fields from phase-modulated delocalized modes that coherently add up to enhance an initial optical hotspot. We therefore develop a simple phenomenological model of the enhancement of the NPL intensity in the center of the wavefront-shaped area.

In brief, we suppose that, before optimization, the electric fields due to delocalized modes in the area of interest have random phases and can be neglected in the initial NPL emitted by the center of the wavefront-shaped area. After optimization, we consider that the electric fields of all the delocalized modes are now in phase and can be coherently added to the initial excitation field impinging on the center of the wavefront-shaped area. To take into account that plasmon polaritons propagating on disordered surfaces are multiply scattered, ${ }^{50}$ we consider that each $i j$ macropixel of the SLM produces a surface cylindrical wave that is exponentially damped because of absorption. With $r_{i j}$ the distance between the center of the considered macropixel and the center of the wavefront-shaped area, the amplitude of the electric field of the damped cylindrical wave in the center of the wavefront-shaped area can be written as $\eta E_{0} e^{-r_{i j} / L} \sqrt{\lambda / 2 \pi r_{i j}}$, with $E_{0}$ the amplitude of the electric field of the wavefront-shaped collimated excitation of wavelength $\lambda=790 \mathrm{~nm}, \eta$ the coupling efficiency of the excitation field to the damped cylindrical wave and $L$ the interaction length of the delocalized modes. ${ }^{33}$ As the size of the SLM macropixels on the sample surface are significantly larger than the nanoscale morphology of the disordered metasurface, we assume that 
an averaging effect leads to similar interaction lengths and coupling efficiencies for all pixels. Importantly, $L$ was estimated experimentally around $5 \mu \mathrm{m}$ in similar samples. ${ }^{33}$ If we define the enhancement factor of the NPL signal $\rho$ as the ratio between optimized and initial intensities, then

$$
\rho=\left|1+\eta \sum_{i j} e^{-r_{i j} / L} \sqrt{\frac{\lambda}{2 \pi r_{i j}}}\right|^{2 p}
$$

where $p=2.8$ is the nonlinear intensity dependence of the luminescence signal (see Fig. S2). Equation 1 assumes that the excitation cross-section of the sample leading to the emission of nonlinear luminescence is the same for a plane-wave excitation or for 2D propagating waves. Since the number and position of pixels are defined by the wavefront-shaped area, this equation provides a simple phenomenological dependence of the average enhancement factors as a function of the size of the wavefront-shaped area. The solid lines in Fig. 3-b correspond to modeled enhancement factors for $L=5 \mu \mathrm{m}$ and $\eta=0.011$ (blue line) and $\eta=0.013$ (green line), respectively. Our phenomenological model therefore agrees qualitatively with a two-order of magnitude enhancement of the NPL intensities using phase-only wavefront shaping in areas larger than $20 \mu \mathrm{m}$. A discrepancy of about one order of magnitude is observed when comparing the experimental data points and the calculated values in Fig. 3-b for phase patterns smaller than $10 \mu \mathrm{m}$, confirming the influence of intensity fluctuations when the size of the wavefront-shaped area is similar to the diffraction limit. Importantly, the coupling efficiency $\eta$ is defined for the amplitude of the electric field. Therefore, the experimental data for large wavefront-shaped areas indicate a coupling efficiency in intensity of about $0.01 \%$. This is significantly lower than typical coupling efficiencies of $\sim 1 \%$ that were estimated for propagating surface plasmons in rough gold surfaces. ${ }^{51}$ This discrepancy can be due to a non-optimum wavefront-shaping process because of the limited number of phases and the fixed number of iterations of the algorithm; but also to a limited number of delocalized modes that are capable of modifying the electric field in the center of the wavefront-shaped area (as already discussed when considering the final phase pattern in Fig. 
$1-d)$.

\section{Conclusion}

By tuning the phase of a far-field excitation when coupling to delocalized plasmonic modes, we were able to optimize near-field optical hotspots in disordered gold metasurfaces by wavefront shaping. This process is performed using a random iterative algorithm and monitored by a typical two-order of magnitude enhancement of the nonlinear photoluminescence of gold in a chosen diffracted-limited area. We demonstrate that this process is the most efficient at the percolation threshold, when the sample exhibits the highest level of morphological complexity and disorder. Importantly, the hypothesis of a phase-only modulation is only valid when the wavefront-shaped area is significantly larger than the diffraction limit. Although intensity fluctuations hinder the quantitative estimation of maximum enhancement values for the nonlinear luminescence signal, our experiments agree qualitatively with a simple phenomenological model using optical parameters of disordered gold films already reported in the literature. Interestingly, this model highlights the important parameters that favor the enhancement of optical hotspots and, therefore, of NPL intensities using wavefront shaping: increasing the interaction length $L$ (by using silver instead of gold ${ }^{33}$ ) and increasing the coupling efficiency $\eta$ to delocalized modes (for instance, by tuning the wettability of the deposited metal in order to increase the thickness and, thus, the scattering cross-section of resonant nanostructures on the metasurface). While these results already open exciting opportunities for the modulation of optical hotspots in complex gold metasurfaces; their extension to a matrix formalism, in order to couple desired near-field properties with a known far-field excitation, would allow the development of fully programmable disordered plasmonic substrates that provide surface-enhanced spectroscopic information from chosen and well-defined areas of a given sample. 


\section{Acknowledgement}

The authors thank A. Goetschy for discussions. This work was supported by the French Direction Générale de l'Armement (DGA) under project 2017706. Work at Institut Langevin was supported by LABEX WIFI (Laboratory of Excellence within the French Program "Investments for the Future") under references ANR-10-LABX-24 and ANR-10-IDEX-0001-02 PSL*. Work at Laboratoire Kastler Brossel was supported by the European Research Coun-

cil (ERC) under project 278025. S.G. is a member of the Institut Universitaire de France (IUF).

\section{Supporting Information Available}

Experimental procedures and complementary measurements of the size of the optimized NPL signal, of the nonlinear dependence of the luminescence of gold with respect to the excitation intensity and of the optimization of the TPF from a homogeneous fluorescent surface due to fluctuations of the excitation intensity.

\section{References}

(1) Vellekoop, I. M.; Mosk, A. P. Focusing coherent light through opaque strongly scattering media. Optics Lett. 2007, 32, 2309-2311.

(2) Popoff, S. M.; Lerosey, G.; Carminati, R.; Fink, M.; Boccara, A. C.; Gigan, S. Measuring the Transmission Matrix in Optics: An Approach to the Study and Control of Light Propagation in Disordered Media. Phys. Rev. Lett. 2010, 104, 100601.

(3) Park, J.-H.; Park, C.; Yu, H.; Park, J.; Han, S.; Shin, J.; Ko, S. H.; Nam, K. T.; Cho, Y.-H.; Park, Y. Subwavelength light focusing using random nanoparticles. Nature Photon. 2013, 7, 454-458. 
(4) Aulbach, J.; Gjonaj, B.; Johnson, P. M.; Mosk, A. P.; Lagendijk, A. Control of Light Transmission through Opaque Scattering Media in Space and Time. Phys. Rev. Lett. 2011, 106, 103901.

(5) Katz, O.; Small, E.; Bromberg, Y.; Silberberg, Y. Focusing and compression of ultrashort pulses through scattering media. Nature Photon. 2011, 5, 372-377.

(6) Mosk, A. P.; Lagendijk, A.; Lerosey, G.; Fink, M. Controlling waves in space and time for imaging and focusing in complex media. Nature Photon. 2012, 6, 283-292.

(7) Mounaix, M.; Andreoli, D.; Defienne, H.; Volpe, G.; Katz, O.; Gresillon, S.; Gigan, S. Spatiotemporal Coherent Control of Light through a Multiple Scattering Medium with the Multispectral Transmission Matrix. Phys. Rev. Lett. 2016, 116, 253901.

(8) Rotter, S.; Gigan, S. Light fields in complex media: Mesoscopic scattering meets wave control. Rev. Mod. Phys. 2017, 89, 015005.

(9) Vellekoop, I. M.; Mosk, A. P. Phase control algorithms for focusing light through turbid media. Opt. Commun. 2008, 281, 3071-3080.

(10) Conkey, D. B.; Brown, A. N.; Caravaca-Aguirre, A. M.; Piestun, R. Genetic algorithm optimization for focusing through turbid media in noisy environments. Opt. Express 2012, 20, 4840-4849.

(11) Boniface, A.; Mounaix, M.; Blochet, B.; Piestun, R.; Gigan, S. Transmission-matrixbased point-spread-function engineering through a complex medium. Optica 2017, 4, $54-59$.

(12) Mounaix, M.; Aguiar, H. B. d.; Gigan, S. Temporal recompression through a scattering medium via a broadband transmission matrix. Optica 2017, 4, 1289-1292.

(13) Dong, J.; Rafayelyan, M.; Krzakala, F.; Gigan, S. Optical Reservoir Computing Using 
Multiple Light Scattering for Chaotic Systems Prediction. IEEE J. Sel. Top. Quantum Electron. 2020, 26, 7701012.

(14) Gresillon, S.; Aigouy, L.; Boccara, A. C.; Rivoal, J. C.; Quelin, X.; Desmarest, C.; Gadenne, P.; Shubin, V. A.; Sarychev, A. K.; Shalaev, V. M. Experimental observation of localized optical excitations in random metal-dielectric films. Phys. Rev. Lett. 1999, 82, 4520-4523.

(15) Li, K. R.; Stockman, M. I.; Bergman, D. J. Self-similar chain of metal nanospheres as an efficient nanolens. Phys. Rev. Lett. 2003, 91, 227402.

(16) Aeschlimann, M.; Bauer, M.; Bayer, D.; Brixner, T.; Abajo, F. J. G. d.; Pfeiffer, W.; Rohmer, M.; Spindler, C.; Steeb, F. Adaptive subwavelength control of nano-optical fields. Nature 2007, 446, 301-304.

(17) Volpe, G.; Cherukulappurath, S.; Juanola Parramon, R.; Molina-Terriza, G.; Quidant, R. Controlling the Optical Near Field of Nanoantennas with Spatial PhaseShaped Beams. Nano Lett. 2009, 9, 3608-3611.

(18) Devilez, A.; Stout, B.; Bonod, N. Mode-balancing far-field control of light localization in nanoantennas. Phys. Rev. B 2010, 81, 245128.

(19) Volpe, G.; Molina-Terriza, G.; Quidant, R. Deterministic Subwavelength Control of Light Confinement in Nanostructures. Phys. Rev. Lett. 2010, 105, 216802.

(20) Gjonaj, B.; Aulbach, J.; Johnson, P. M.; Mosk, A. P.; Kuipers, L.; Lagendijk, A. Active spatial control of plasmonic fields. Nature Photon. 2011, 5, 360-363.

(21) Kao, T. S.; Jenkins, S. D.; Ruostekoski, J.; Zheludev, N. I. Coherent Control of Nanoscale Light Localization in Metamaterial: Creating and Positioning Isolated Subwavelength Energy Hot Spots. Phys. Rev. Lett. 2011, 106, 085501. 
(22) Kao, T. S.; Rogers, E. T. F.; Ou, J. Y.; Zheludev, N. I. "Digitally" Addressable Focusing of Light into a Subwavelength Hot Spot. Nano Lett. 2012, 12, 2728-2731.

(23) Veksler, D.; Maguid, E.; Shitrit, N.; Ozeri, D.; Kleiner, V.; Hasman, E. Multiple Wavefront Shaping by Metasurface Based on Mixed Random Antenna Groups. ACS Photonics 2015, 2, 661-667.

(24) Zhang, Y.; Demesy, G.; Haggui, M.; Gérard, D.; Béal, J.; Dodson, S.; Xiong, Q.; Plain, J.; Bonod, N.; Bachelot, R. Nanoscale Switching of Near-Infrared Hot Spots in Plasmonic Oligomers Probed by Two-Photon Absorption in Photopolymers. ACS Photonics 2018, 5, 918-928.

(25) Stockman, M. I.; Faleev, S. V.; Bergman, D. J. Localization versus Delocalization of Surface Plasmons in Nanosystems: Can One State Have Both Characteristics? Phys. Rev. Lett. 2001, 87, 167401.

(26) Seal, K.; Genov, D. A.; Sarychev, A. K.; Noh, H.; Shalaev, V. M.; Ying, Z. C.; Zhang, X.; Cao, H. Coexistence of Localized and Delocalized Surface Plasmon Modes in Percolating Metal Films. Phys. Rev. Lett. 2006, 97, 206103.

(27) Krachmalnicoff, V.; Castanie, E.; De Wilde, Y.; Carminati, R. Fluctuations of the Local Density of States Probe Localized Surface Plasmons on Disordered Metal Films. Phys. Rev. Lett. 2010, 105, 183901.

(28) Castanie, E.; Krachmalnicoff, V.; Caze, A.; Pierrat, R.; De Wilde, Y.; Carminati, R. Distance dependence of the local density of states in the near field of a disordered plasmonic film. Opt. Lett. 2012, 37, 3006-3008.

(29) Awada, C.; Barbillon, G.; Charra, F.; Douillard, L.; Greffet, J.-J. Experimental study of hot spots in gold/glass nanocomposite films by photoemission electron microscopy. Phys. Rev. B 2012, 85, 045438. 
(30) Gunn, J. M.; Ewald, M.; Dantus, M. Polarization and Phase Control of Remote SurfacePlasmon-Mediated Two-Photon-Induced Emission and Waveguiding. Nano Lett. 2006, 6, 2804-2809.

(31) Gunn, J. M.; High, S. H.; Lozovoy, V. V.; Dantus, M. Measurement and Control of Ultrashort Optical Pulse Propagation in Metal Nanoparticle-Covered Dielectric Surfaces. J. Phys. Chem. C 2010, 114, 12375-12381.

(32) Aeschlimann, M.; Brixner, T.; Cunovic, S.; Fischer, A.; Melchior, P.; Pfeiffer, W.; Rohmer, M.; Schneider, C.; Strüber, C.; Tuchscherer, P.; et al., Nano-Optical Control of Hot-Spot Field Superenhancement on a Corrugated Silver Surface. IEEE J. Sel. Top. Quantum Electron. 2012, 18, 275-282.

(33) Bondareff, P.; Volpe, G.; Gigan, S.; Gresillon, S. Probing Extended Modes on Disordered Plasmonic Networks by Wavefront Shaping. ACS Photonics 2015, 2, 1658-1662.

(34) Gopinath, A.; Boriskina, S. V.; Reinhard, B. M.; Negro, L. D. Deterministic aperiodic arrays of metal nanoparticles for surface-enhanced Raman scattering (SERS). Opt. Express 2009, 17, 3741-3753.

(35) Savo, S.; Papasimakis, N.; Zheludev, N. I. Localization of electromagnetic fields in disordered metamaterials. Phys. Rev. B 2012, 85, 121104.

(36) Voss, R. F.; Laibowitz, R. B.; Allessandrini, E. I. Fractal (Scaling) Clusters in Thin Gold Films near the Percolation Threshold. Phys. Rev. Lett. 1982, 49, 1441-1444.

(37) Moskovits, M. Surface roughness and the enhanced intensity of Raman scattering by molecules adsorbed on metals. J. Chem. Phys. 1978, 69, 4159-4161.

(38) Hartstein, A.; Kirtley, J. R.; Tsang, J. C. Enhancement of the Infrared Absorption from Molecular Monolayers with Thin Metal Overlayers. Phys. Rev. Lett. 1980, 45, $201-204$. 
(39) Biteen, J. S.; Pacifici, D.; Lewis, N. S.; Atwater, H. A. Enhanced Radiative Emission Rate and Quantum Efficiency in Coupled Silicon Nanocrystal-Nanostructured Gold Emitters. Nano Lett. 2005, 5, 1768-1773.

(40) Gadenne, P.; Rivoal, J. C. In Optical Properties of Nanostructured Random Media; Shalaev, V. M., Ed.; Springer Berlin Heidelberg, 2002; p 187-215.

(41) Gopinath, A.; Boriskina, S. V.; Feng, N.-N.; Reinhard, B. M.; Negro, L. D. PhotonicPlasmonic Scattering Resonances in Deterministic Aperiodic Structures. Nano Lett. 2008, 8, 2423-2431.

(42) Beversluis, M. R.; Bouhelier, A.; Novotny, L. Continuum generation from single gold nanostructures through near-field mediated intraband transitions. Phys. Rev. B 2003, $68,115433$.

(43) Haug, T.; Klemm, P.; Bange, S.; Lupton, J. M. Hot-Electron Intraband Luminescence from Single Hot Spots in Noble-Metal Nanoparticle Films. Phys. Rev. Lett. 2015, 115, 067403.

(44) Molinaro, C.; El Harfouch, Y.; Palleau, E.; Eloi, F.; Marguet, S.; Douillard, L.; Charra, F.; Fiorini-Debuisschert, C. Two-Photon Luminescence of Single Colloidal Gold Nanorods: Revealing the Origin of Plasmon Relaxation in Small Nanocrystals. J. Phys. Chem. C 2016, 120, 23136-23143.

(45) Ghenuche, P.; Cherukulappurath, S.; Taminiau, T. H.; van Hulst, N. F.; Quidant, R. Spectroscopic Mode Mapping of Resonant Plasmon Nanoantennas. Phys. Rev. Lett. 2008, 101, 116805 .

(46) Michaels, A. M.; Jiang, J.; Brus, L. Ag Nanocrystal Junctions as the Site for SurfaceEnhanced Raman Scattering of Single Rhodamine 6G Molecules. J. Phys. Chem. B 2000, 104, 11965-11971. 
(47) Maier, S. A.; Kik, P. G.; Atwater, H. A.; Meltzer, S.; Harel, E.; Koel, B. E.; Requicha, A. A. G. Local detection of electromagnetic energy transport below the diffraction limit in metal nanoparticle plasmon waveguides. Nat. Mater. 2003, 2, 229-232.

(48) Koenderink, A. F.; Polman, A. Complex response and polariton-like dispersion splitting in periodic metal nanoparticle chains. Phys. Rev. B 2006, 74, 033402.

(49) de Waele, R.; Koenderink, A. F.; Polman, A. Tunable nanoscale localization of energy on plasmon particle arrays. Nano Lett. 2007, 7, 2004-2008.

(50) Akkermans, E.; Montambaux, G. Mesoscopic Physics of Electrons and Photons; Cambridge University Press, 2007.

(51) Salomon, L.; Bassou, G.; Aourag, H.; Dufour, J. P.; de Fornel, F.; Carcenac, F.; Zayats, A. V. Local excitation of surface plasmon polaritons at discontinuities of a metal film: Theoretical analysis and optical near-field measurements. Phys. Rev. B 2002, 65, 125409. 
TOC graphic

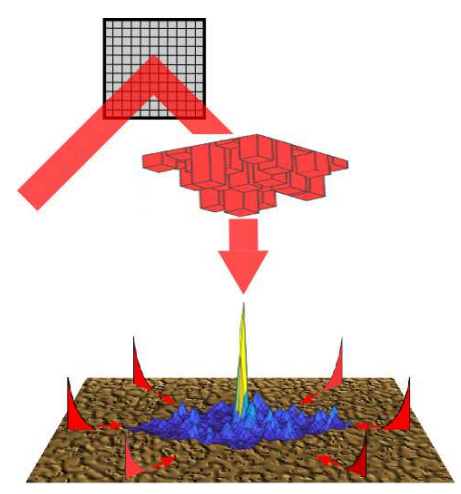

